\title{
Editorial
}

\section{Internet of Everything}

\author{
Laurence T. Yang, ${ }^{1}$ Beniamino Di Martino, ${ }^{2}$ and Qingchen Zhang ${ }^{1}$ \\ ${ }^{1}$ St. Francis Xavier University, Antigonish, NS, Canada \\ ${ }^{2}$ University of Campania "Luigi Vanvitelli", Caserta, Italy \\ Correspondence should be addressed to Laurence T. Yang; ltyang@stfx.ca
}

Received 16 May 2017; Accepted 16 May 2017; Published 3 July 2017

Copyright (c) 2017 Laurence T. Yang et al. This is an open access article distributed under the Creative Commons Attribution License, which permits unrestricted use, distribution, and reproduction in any medium, provided the original work is properly cited.

Due to recent advancements in big data, connection technologies, and smart devices, our environment is transforming into an "Internet of Everything" (IoE). The Internet of Everything has become a catch-all phrase to describe adding connectivity and intelligence to just about every device in order to give them special functions. However, this can be quite reductive, as IoE provides links among not only things, but also data, people, and (business) processes. Evolution of current sensor and device networks, with strong interaction with people and social environments, will have a dramatic impact on everything from city planning, first responders, military, and health. Several Internet and connection-based paradigms fall under the IoE umbrella, such as Internet of Things (IoT), Internet of People (IoP), and Industrial Internet (II). While such areas cover many aspects of today's life, there is still the strong requirement to contextualize and integrate data and information coming from different networks and frameworks. Indeed, there is a need to provide a common ground for integrating information coming from heterogeneous sources. Such a shared ecosystem would allow for the interaction among data, sensor inputs, and heterogeneous systems.

Therefore, it is high time to call for contributions to further stimulate the continuing efforts to enable such an integrated framework; some fundamental issues are required to be addressed to provide the necessary bridge between different data representations and to solve terminology incongruence. This special issue attracted numerous submissions, out of which twenty papers have been accepted to be published. These accepted papers are expected to highlight some of the important aspects outlined above. It is the pleasure for the guest editorial team to introduce these papers as follows.

The paper entitled "Implementation and Optimization of GPU-Based Static State Security Analysis in Power Systems" by Y. Chen et al. contributes to static state security in power systems. A sensitivity analysis-based method with graphics processing unit (GPU) is proposed for power systems, which involves load flow analysis and sensitivity analysis. A multifrontal method for sparse LU factorization is explored on GPU in the load flow analysis while the varying matrix operations during sensitivity analysis on GPU are highly optimized.

The paper entitled "RAID-6Plus: A Comprised Methodology for Extending RAID-6 Codes" by M.-Z. Deng et al. focuses on RAID-6 code. In particular, a new RAID6 code extending methodology with shorter reconstruction window is developed in this study, which provides a balanced tradeoff of flexible reliability and better system performance. Furthermore, an example extension code called RDP+ is presented based on RDP in terms of encoding and single failure reconstruction improvement. In order to validate and evaluate the presented extending methodology, a new metric called Q-metric is proposed.

In the paper entitled "Recommending Locations Based on Users' Periodic Behaviors" by B. Xu et al., the challenging topic of location recommendation based on user's life behavior is addressed. In view of multiple periodic behaviors existing in time series, an algorithm which can mine all periods in time series is proposed in this paper. Particularly, 
locations using item-based collaborative filtering algorithm are recommended based on the periodic behaviors.

The paper entitled "Recovering Individual's Commute Routes Based on Mobile Phone Data" by X. Song et al., aims to propose a commute routes recovering model to recover individuals' commute routes based on passively generated mobile phone data, which applies two modules. The first is data preprocessing module, which extracts commute trajectories from raw dataset and formats the road network into a better modality. The second module combines two kinds of information together and generates the commute route with the highest possibility.

The paper entitled "A Process Mining Based Service Composition Approach for Mobile Information Systems" by C. Huang et al. investigates the connection between large scale of data and the associated business processes in the Internet of Everything (IoE) environment. Particularly, a process mining based service composition approach is proposed in this paper in order to improve the adaptiveness and efficiency of compositions. Firstly, a preprocessing is conducted to extract existing service execution information from server-side logs. Then process mining algorithms are applied to discover the overall event sequence with preprocessed data.

The paper entitled "Energy-Efficient Broadcasting Scheme for Smart Industrial Wireless Sensor Networks" by Z. Chen et al. proposes a novel energy-efficient broadcast scheme with adjustable broadcasting radius to improve the performance of network upgrade in smart industrial wireless sensor networks. In their scheme, the nonhotspots sensor nodes take full advantage of their residual energy caused in data collection period to improve the packet reception probability and reduce the broadcasting delay of code packet transmission by enlarging the broadcasting radius, that is, the transmitting power.

The paper entitled "Exploiting Wireless Received Signal Strength Indicators to Detect Evil-Twin Attacks in Smart Homes" by Z. Tang et al. focuses on Evil-Twin attack in smart home environments. In particular, this paper presents a novel Evil-Twin attack detection method based on the received signal strength indicator (RSSI). This approach considers the RSSI as a fingerprint of APs and uses the fingerprint of the genuine AP to identify fake ones. Furthermore, two schemes are presented to detect a fake AP in two different scenarios where the genuine AP can be located at either single or multiple locations in the property, by exploiting the multipath effect of the Wi-Fi signal.

In the paper entitled "An Extended Technology Acceptance Model for Mobile Social Gaming Service Popularity Analysis" by H. Chen et al., an empirical study on WeChat, China's most popular mobile social network, is presented. Particularly, a technology acceptance model (TAM) is applied to study the reasons beneath the popularity of games in mobile social networks. Furthermore, factors from social and mobile perspective are incorporated into the conventional TAM and their influence and relationships are studied.

The paper entitled "Power-Aware Resource Reconfiguration Using Genetic Algorithm in Cloud Computing" by L. Deng et al. aims to address the power-aware resource reconfiguration in cloud computing. In this paper, several algorithms for VM placement (multiobjective genetic algorithm (MOGA), power-aware multiobjective genetic algorithm (pMOGA), and enhanced power-aware multiobjective genetic algorithm (EpMOGA)) are presented to improve stability of VM placement pattern with less migration overhead. Furthermore, nondominated sorting genetic algorithm II (NSGAII) is used to select new generations during evolution process.

The paper entitled "Delay-Aware Program Codes Dissemination Scheme in Internet of Everything" by Y. Xuan et al. investigates a delay-aware program dissemination (DAPD) scheme to disseminate program codes with fast, reliable, and energy-efficient style. DAPD scheme improves the performance of bulk codes dissemination through the following two aspects. (1) Due to the fact that a high transmitting power can significantly improve the quality of wireless links, transmitting power of sensors with more residual energy is enhanced to improve link quality. (2) Due to the fact that performance of correlated dissemination tends to degrade in a highly dynamic environment, link correlation is autonomously updated in DAPD during codes dissemination to maintain improvements brought by correlated dissemination.

The paper entitled "Channel Selection Policy in Multi-SU and Multi-PU Cognitive Radio Networks with Energy Harvesting for Internet of Everything" by F. Hu et al. contributes to channel selection in a multi-SU and multi-PU cognitive radio network. In this paper, the authors adopt cooperative sensing method to avoid the packet collision between SUs and PUs and focus on how to collect the spectrum sensing data of SUs for cooperative sensing. Furthermore, they propose a competitive set based channel selection policy for multiSU where all SUs competing for data transmission or energy harvesting in the same channel will form a competitive set.

The paper entitled "Making Image More Energy Efficient for OLED Smart Devices" by D. Li et al. focuses on energy consumption of OLED displaying in smart devices. In particular, this paper proposes an approach to improve image energy efficiency on OLED displays by perceiving image content. The key idea of this approach is to eliminate undesired details while preserving the region of interest of the image by leveraging the color and spatial information. First, we use edge detection algorithm to extract region of interest (ROI) of an image.

In the paper entitled "Speed-Density Model of Interrupted Traffic Flow Based on Coil Data" by C. Yu et al., a new method which can accurately describe the speed-density relation of interrupted traffic flow is proposed for speed fluctuation characteristics. The model of upper and lower bounds of critical values obtained by fitting the data of the coils on urban roads can accurately and intuitively describe the state of urban road traffic, and the physical meaning of each parameter plays an important role in the prediction and analysis of such traffic.

The paper entitled "A Novel Exercise Thermophysiology Comfort Prediction Model with Fuzzy Logic" by N. Jia et al. aims to address the prediction of exercise accidents in a regular exercise program. Particularly, a human thermophysiology regulatory model is designed to enhance the human thermophysiology simulation in the HCE system. 
Some important thermal and physiological performances can be simulated. According to the simulation results, a human exercise thermophysiology comfort prediction method based on fuzzy inference system is proposed.

The paper entitled "Time-Aware IoE Service Recommendation on Sparse Data" by L. Qi et al. investigates the challenges of the recommendation technique on the service selection decision of target users. In view of the challenges, a time-aware service recommendation approach is proposed in this paper. Concretely, the time-aware user similarity is first calculated; afterwards, indirect friends of the target user are inferred by Social Balance Theory (e.g., "enemy's enemy is a friend" rule); finally, the services preferred by indirect friends of the target user are recommended to the target user.

The paper entitled "Congestion Control Mechanism for Intermittently Connected Wireless Network" by R. Wang et al. proposes a congestion control mechanism that is based on the network state dynamic perception. Specifically, through estimating the congestion risk when a node receives packets, ICWN can reduce the probability of becoming congested. Moreover, due to ICWN's network dynamics, the congestion risk threshold is determined by jointly taking into account the average packet size, average forwarding risk, and available buffer resources. Further, the service ability of a node in a distributed manner is evaluated by integrating the recommendation information from other intermediate nodes. Additionally, a node is selected as a relay node according to both the congestion risk and service ability.

The paper entitled "STLIS: A Scalable Two-Level Index Scheme for Big Data in IoT" by Y. Leng et al. focuses on index scheme for big data in IoT. In particular, this paper proposes a scalable two-level index scheme (STLIS) for RDF data. In the first level, a compressed path template tree (CPTT) index is proposed based on S-tree to retrieve the candidate sets of full path. In the second level, a hierarchical edge index (HEI) and a node-predicate (NP) index are created to accelerate the match.

In the paper entitled "An Indoor Ultrasonic Positioning System Based on TOA for Internet of Things" by J. Li et al., an ultrasonic indoor positioning system is presented, which can achieve centimeter-level precise positioning of objects moving indoors. The system is based on long-baseline positioning technology that uses code division multiplexing access mechanism. Particularly, the system uses wideband pseudorandom noise signal called Gold sequences for multiuser identification and slant range measurement.

The paper entitled "Phase Clustering Based Modulation Classification Algorithm for PSK Signal over Wireless Environment" by Q. An et al. proposes a new signal classifier for phase shift keying (PSK) signals. The periodicity of signal's phase is utilized as the assorted character, with which a fractional function is constituted for phase clustering. Particularly, an advanced estimator is proposed for estimating the frequency offset and balancing estimation accuracy and range under low signal-to-noise ratio (SNR) conditions.

The paper entitled "A High-Order CFS Algorithm for Clustering Big Data” by F. Bu et al. investigates the clustering scheme for big data in Internet of Things. In particular, this paper proposes a high-order CFS algorithm (HOCFS) to cluster heterogeneous data by combining the CFS clustering algorithm and the dropout deep learning model, whose functionality rests on three pillars: (i) an adaptive dropout deep learning model to learn features from each type of data, (ii) a feature tensor model to capture the correlations of heterogeneous data, and (iii) a tensor distance-based highorder CFS algorithm to cluster heterogeneous data.

\section{Acknowledgments}

The guest editorial team would like to thank all the authors for submitting their manuscripts to this special issue and all the invited reviewers for their time and constructive feedback.

Laurence T. Yang

Beniamino Di Martino

Qingchen Zhang 

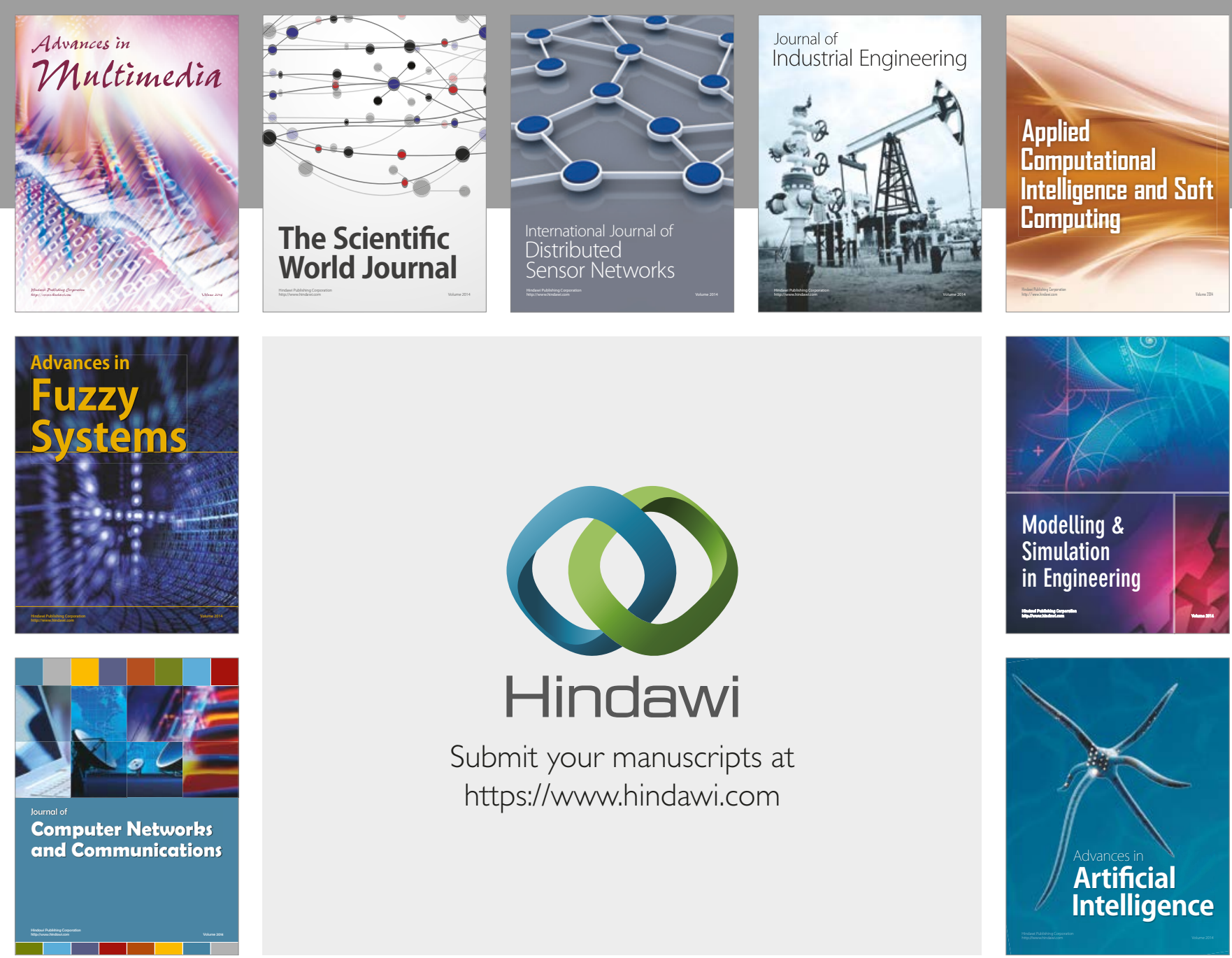

\section{Hindawi}

Submit your manuscripts at

https://www.hindawi.com
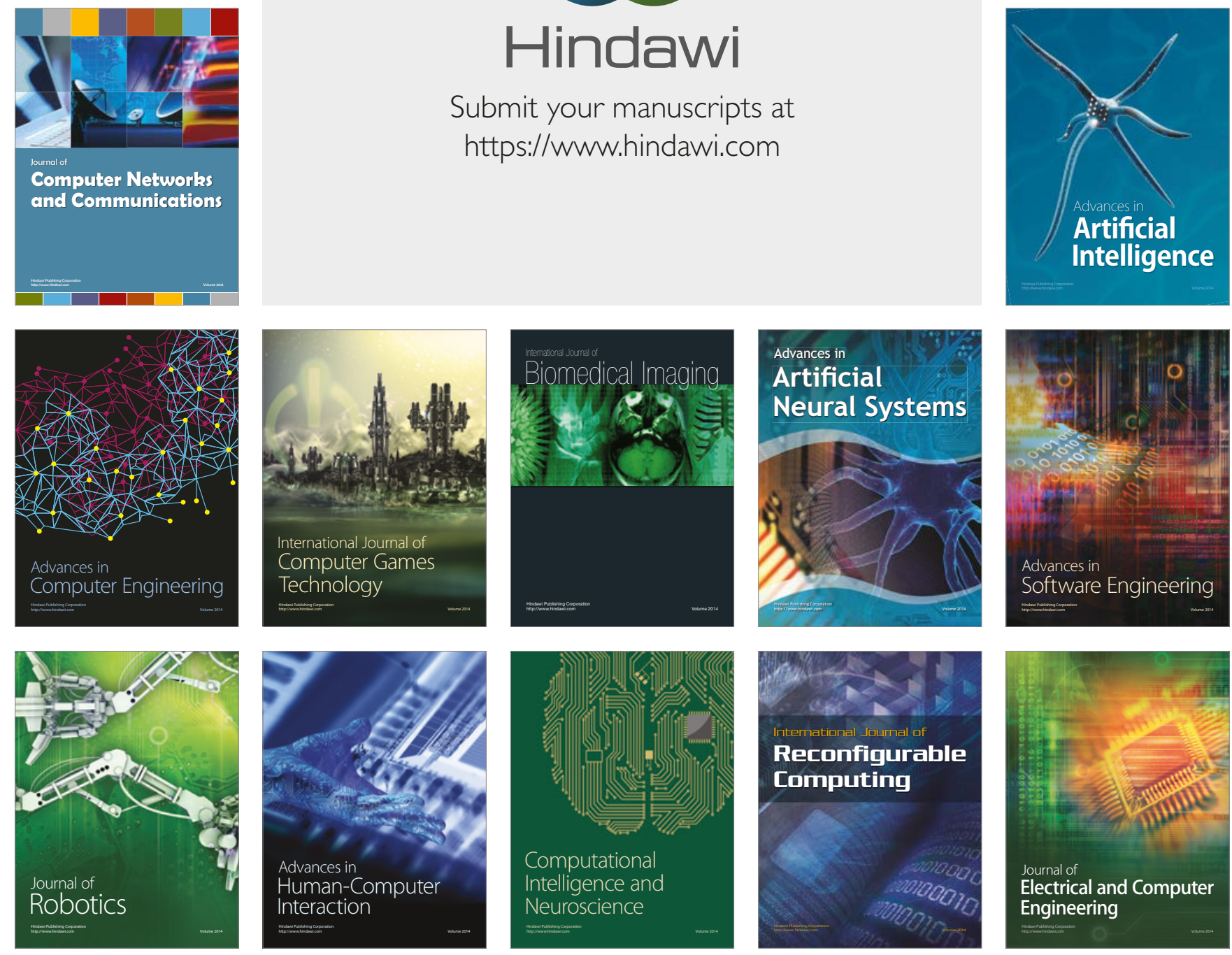